Results From October 2012 through November 2015, 180 subjects were treated at 26 sites. 36-month follow up, $94.8 \%$ (127/134) completed clinical follow-up, 76.9\% (103/134) completed clinical and angiogram follow-up. The functional mRS scores remained unchanged or improved in most subjects at 36 months $(85.0 \%, 108 / 127)$ when compared to the same measure at baseline. The rate of Raymond 1 total occlusion continued to improve at 36-month follow up compared to 12-month scores; $79.0 \%(83 / 105)$ of subjects demonstrated Raymond 1 occlusion at 36 months vs. $66.1 \%(119 / 180)$ at 12 months. Raymond 2 occlusion at 36 months was $20.0 \%$ (21/105), compared to $9.4 \%(17 / 180)$ of subjects with Raymond 2 at 12 months. The Raymond 3 occlusion rate decreased to $1.0 \%(1 / 105)$ of subjects at 36 months compared to $15.0 \%(27 / 180) 12$-months post procedure. The proportion of subjects experiencing new or worsening major ipsilateral stroke, as adjudicated by the CEC, was $8.3 \%(15 / 180)$ at 12 months and increased to $8.8 \%(16 / 180)$ and $9.4 \%(17 / 180)$ subjects at 24 , and 36 months respectively. Four $(2.2 \%, 4 /$ 180) experienced aneurysm rupture by 12 months, all within the first-week post target aneurysm treatment. No additional late aneurysm ruptures occurred through 36 months. Four subjects who received retreatment beyond 12 months had a residual aneurysm. The 60-month occlusion durability and safety endpoint results will be the subject of a future presentation.

Disclosures R. Hanel: 1; C; Stryker, Medtronic, MicroVention, Balt. 2; C; Stryker, Medtronic, Balt, MicroVention, Phenox, Three Rivers Medical, Elum, Corindus, Shape Medical. 4; C; elum, Three Rivers Medical, Corindus, Cerebrotech, Synchron,
BlinkTBI, Scientia, RIST, InNeuroCo. A. Coon: 2; C; stryker, medtronic, microvention, inneuroco. P. Kan: 2; C; Inneuroco. A. Wakhloo: 1; C; Phillips. 2; C; Stryker, Phenox. 4; C; Inneuroco, EPIEP, Neural Analytics. P. Meyers: 2; C; Stryker.

\section{0-012 ANTI-THROMBOGENIC COATING FOR FLOW DIVERTERS: USING HIGH-FREQUENCY OPTICAL COHERENCE TOMOGRAPHY TO IMAGE ACUTE THROMBUS BURDEN}

R King*, E Langan, M Marosfoi, G Ughi, C Raskett, M Gounis. Radiology, University of Massachusetts, Worcester, MA

\subsection{6/neurintsurg-2019-SNIS.12}

Introduction The use of stents in the treatment of wide neck aneurysms, and more recently the adoption of flow diverters, are important tools for the treatment of aneurysms. ${ }^{1}$ One major complication of such implants is thromboembolism, requiring the use of dual anti-platelet therapy (DAPT). However, due to variable response and inherent risks of DAPT, new stents are being developed with anti-thrombogenic coatings with the goal to reduce or eliminate the need for DAPT. Methods A novel hydrophilic polymer coating (HPC) applied to a nitinol substrate has been shown to resist platelet aggregation in vitro. $^{2}$ We sought to demonstrate preliminary evidence to confirm this observation in vivo. Three pigs were used with different regimens: no antiplatelet medication (NAPT), $81 \mathrm{mg}$ aspirin (SAPT), and $81 \mathrm{mg}$ aspirin with 75 mg Clopidogrel (DAPT). Two control and two coated devices were implanted in each animal.

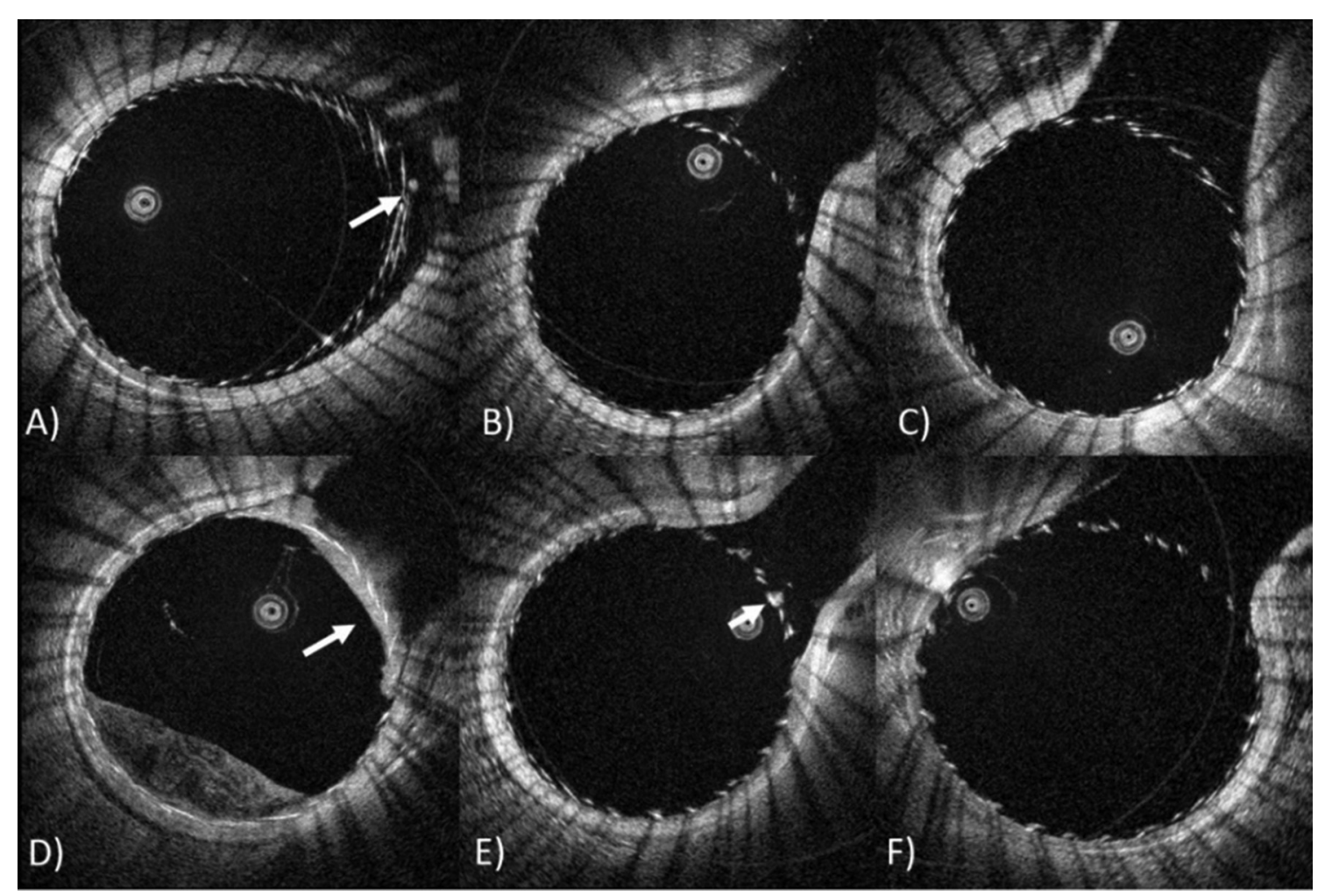

Abstract 0-012 Figure 1 HF-OCT cross-sectional imaging from each animal. Metallic flow-diverter struts are visualized by HF-OCT as small bright objects followed by a shadow. Top row (A-C) shows the coated device, with NAPT (A), SAPT (B) and DAPT (C). There is a small amount of clot (white arrow, A), but once SAPT is added, there is almost none left. Bottom row (D-F) shows the control device, with a large amount of clot with NAPT (white arrow D), requiring DAPT to remove all clot. In image (D), blood residuals due to incomplete artery clearance by contrast injection are visible approximately between 5 and 9 o'clock 
High Frequency Optical Coherence Tomography (HF-OCT), with a spatial resolution approaching 10 microns, was used to study acute thrombus formation along the surface of the device. HF-OCT acquisitions were acquired 20 minutes following device implant. Specifically, the total clot volume on each side branch opening ( $\mathrm{SBO}$ ) was segmented with manual input by a blinded user. Statistical analyses were performed to determine the relative benefit of the HPC coating and the antiplatelet regiment for prevention of platelet aggregation, a linear model was constructed to interrogate the relative importance.

Results The figure 1 shows one section from each device type. It was found that device type and aspirin where significantly correlated with thrombus volume over SBOs. Mean thrombus volume per $\mathrm{mm}^{2}$ of $\mathrm{SBO}$ for coated versus control devices was $0.00033 \mathrm{~mm}$ versus $0.087 \mathrm{~mm}$, respectively $(\mathrm{p}=0.005)$. Mean clot per SBO was $0.004 \mathrm{~mm}$ versus $0.15 \mathrm{~mm}$ in animals receiving aspirin versus NAPT $(\mathrm{p}<0.001)$. The linear model found that mean thrombus $(0.222 \mathrm{~mm})$ was dramatically reduced by the HPC coating (coefficient $=-0.221$ ), aspirin use (coefficient $=-0.214$ ), and that the combined effect removed nearly all clot.

Conclusions This preliminary evidence shows the dramatic effect that the coating can have on the acute thrombus formation. Further, this novel HF-OCT technology allows for quantitative measurements of the amount of thrombus formed on the surface of a flow diverter, not just the presence. Finally, this study shows the possibility that the combined effect of aspirin and this new coating may be sufficient antiplatelet therapy.

\section{REFERENCES}

1. Neurosurgery $2017 ; 80: 40-48$.

2. CardioVasc Intervent Radiol 2018;41:1779-1785.

Disclosures R. King: None. E. Langan: None. M. Marosfoi: None. G. Ughi: 4; C; Gentuity LLC. 5; C; Gentuity LLC. C. Raskett: None. M. Gounis: 2; C; Phenox

\section{0-013 FLOW-DIVERTER BIOLOGICAL IMPROVEMENT WITH CD31 BIOMIMETIC: PRECLINICAL EXPERIENCE}

${ }^{1} \mathrm{~J}$ Cortese ${ }^{*},{ }^{2} \mathrm{C}$ Rasser, ${ }^{2} \mathrm{G}$ Even, ${ }^{2} \mathrm{C}$ Choqueux, ${ }^{3} \mathrm{~S}$ Bardet, ${ }^{1} \mathrm{~J}$ Caroff, ${ }^{1} \mathrm{~L}$ Spelle, ${ }^{2} \mathrm{G}$ Caligiuri, ${ }^{4} \mathrm{~A}$ Rouchaud. ${ }^{1}$ NeuroRadiologie Interventionnelle, CHU BICETRE AP-HP, Le Kremlin Bicetre, France; ${ }^{2}$ CHU Xavier Bichat, INSERM U1148, Paris, France; ${ }^{3}$ Xlim - CNRS, Université de Limoges, Limoges, France; ${ }^{4}$ NeuroRadiologie Interventionnelle, CHU de Limoges, Limoges, France

10.1136/neurintsurg-2019-SNIS.13

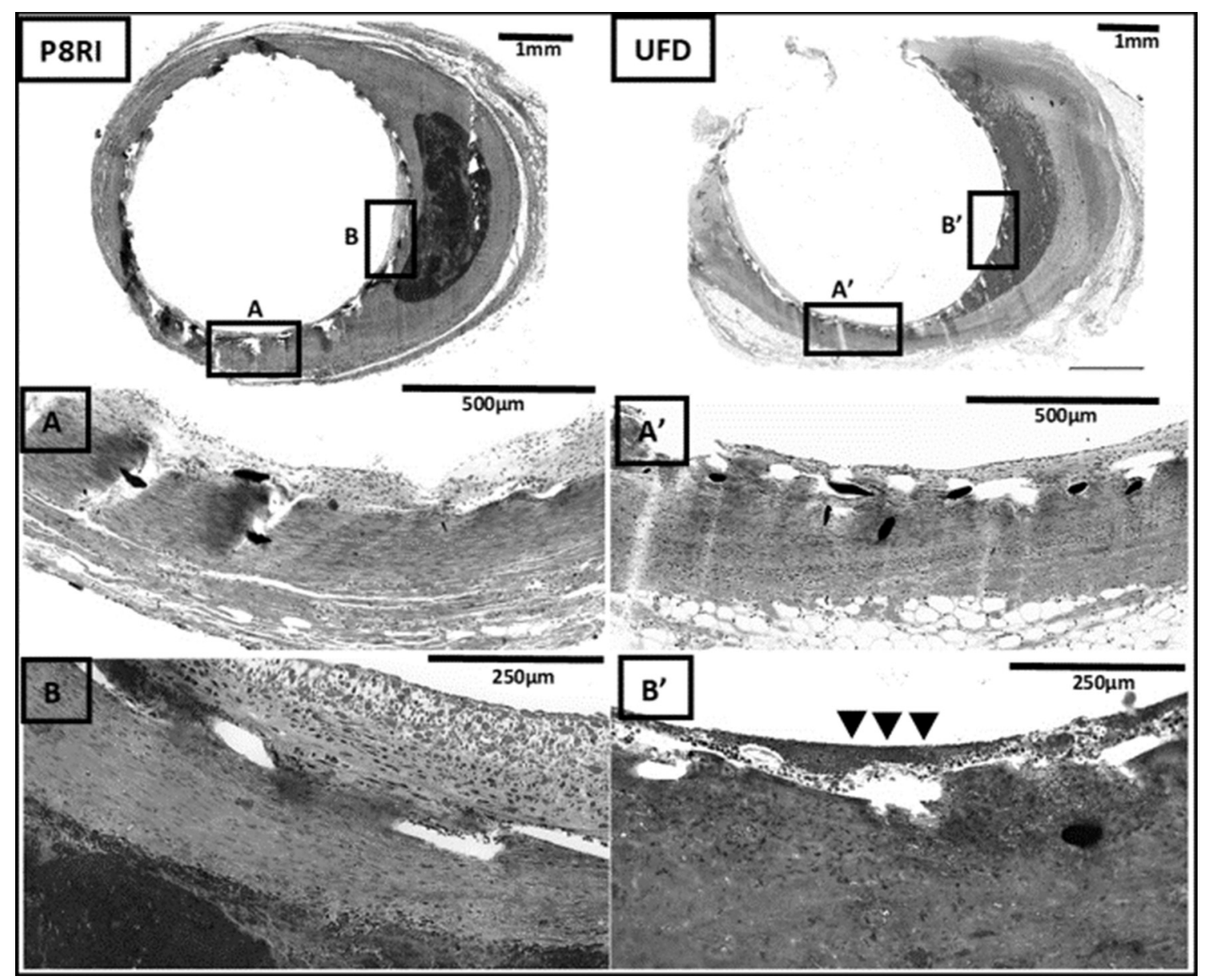

Abstract 0-013 Figure 1 Histological sections of implanted Silk Vista Baby\&reg; Flow-diverters stained with Masson's trichrome. Blue: connective tissue. Pink: cytoplasm. Purple: nuclei. Left column/P8RI: P8RI-coated FD. Right column/UFD: bare/unmodified DF. A, A': details of the arterial wall far from the aneurysm. B, B': detail of the neo-tissue surrounding the stent struts in front of the aneurysm. The tissue that covers the struts on the P8RI-coated DF is formed by continuous endothelium and is supported by a normal media rich in smooth muscle cells, contrary to that of the unmodified FD. Moreover, the tissue that covers the stent struts on the bare FD is distinctly disorganized, poor in extra-cellular component such as collagen and discontinuous. The presence of red blood cells indicates haemorrhage in contact with the stent (black arrow head) 